\title{
ELECTRON-BEAM PROCESSED SAW DEVICES FOR SENSOR APPLICATIONS
}

\author{
Marc Loschonsky ${ }^{1}$,David Eisele ${ }^{1}$, Jeremy Masson ${ }^{2}$, Sébastien Alzuaga ${ }^{3}$, Armin Dadgar $^{4}$, \\ Sylvain Ballandras ${ }^{3}$, and Leonhard Reindl ${ }^{1}$ \\ ${ }^{1}$ Department of Microsystem Engineering (IMTEK), University of Freiburg, Freiburg, Germany. \\ e-mail: loc@imtek.de, eisele@imtek.de, reindl@imtek.de \\ ${ }^{2}$ Senseor, Sophia-Antipolis, France. \\ e-mail: jeremy.masson@senseor.com \\ ${ }^{3}$ FEMTO-ST, Besançon, France. \\ e-mail: sebastien.alzuaga@femto-st.fr, sylvain.ballandras@femto-st.fr \\ ${ }^{4}$ Azzurro Semiconductors AG, Magdeburg, Germany. \\ e-mail: armin.dadgar@azzurro-semiconductors.com
}

\begin{abstract}
In this paper, electron-beam (e-beam) lithography for processing of surface acoustic wave devices is investigated, and its suitability for large-scale processing discussed. Electron-beam lithography is used for exposure of surface acoustic wave (SAW) resonator patterns on polymethyl methacrylate (PMMA) coated piezoelectric substrates. Electron-beam lithography can be used for high frequency SAW designs, due to a minimal finger width of $100 \mathrm{~nm}$ to $400 \mathrm{~nm}$. Such SAW devices can be used for high-frequency sensor applications. This contribution will consider processing, on-wafer characterization, and characterization of sensor effects in instrumentation applications.
\end{abstract}

Index Terms - Electron beam lithography, surface acoustic waves, sensors, high frequency

\section{INTRODUCTION}

In recent years, e-beam lithography is being used to rapidly prototype MEMS devices [1]. On one hand, it offers very high resolution up to $100 \mathrm{~nm}$, using direct resist lithography, without the need of an intermediate mask. On the other hand, e-beam lithography is a sequential method, whereas the e-beam writes the image analogous to the scanning performed inside a CRT display [2],[3]. Consequently, the writing speed of ebeam lithography is in general too slow to be used for large-scale, high-volume processing. But this technology offers the possibility to create SAW devices with user defined electrodes, which were not possible by conventional lithography technology, and could be used for high quality devices, like in space applications.

For high-frequency SAW sensor applications, resonators are commonly used, permitting: very small chip sizes and therefore small packaged sensor elements, the possibility to use shear waves, and also high resolution, due to the high frequency. One promising substrate material for the SAW resonators is a-plane gallium nitride $(\mathrm{GaN})$ on top of r-plane sapphire, permitting shear polarized waves using a-plane $\mathrm{GaN}$, and permitting high wave velocity using sapphire [4]-[5].

\section{DESIGN OF SAW RESONATOR}

The GaN-sapphire wafer used, had a film thickness of $1000 \mathrm{~nm}$. The acoustic wavelength was chosen to be $1200 \mathrm{~nm}$ so that it would be on the same scale, therefore the wave excitation would be mainly inside the piezoelectric film. The mark to pitch ratio (metallization ratio $\mathrm{a} / \mathrm{p}$ ) was chosen to be $60 \%$, resulting in finger widths of $360 \mathrm{~nm}$ and a resonance frequency in the vicinity of $3 \mathrm{GHz}$.

By using simulations of the harmonic admittance of aluminium inter digital transducers (IDT) on a GaN/ sapphire substrate, the design was optimized for an aperture of $30 \mu \mathrm{m}$, an acoustic length of the synchronous resonator of $200 \mu \mathrm{m}$, and $100 \mathrm{~nm}$ aluminum used as an electrode material.

The inter digital transducer (IDT) was designed with 70 finger pairs in the middle, and 95 reflector fingers on both the left and right side of the IDT. A schematic of the SAW resonator is shown in Figure 1. 


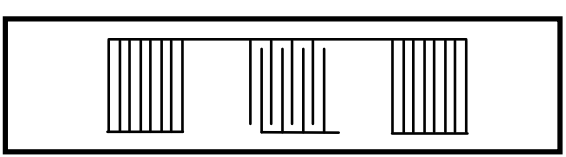

Figure 1. Scheme of the synchronous SAW resonator with 70 IDT finger pairs in the middle, and 95 reflector fingers on both the left and the right side.

\section{ELECTRON-BEAM PROCESSING}

The processing was achieved using an e-beam lithography system for rapid prototyping. The exposing window of the system was $200 \times 200 \mu \mathrm{m}^{2}$ using an ebeam dose of approximately $130 \mu \mathrm{C} / \mathrm{cm}^{2}$. The basic process steps are shown in Figure 2.

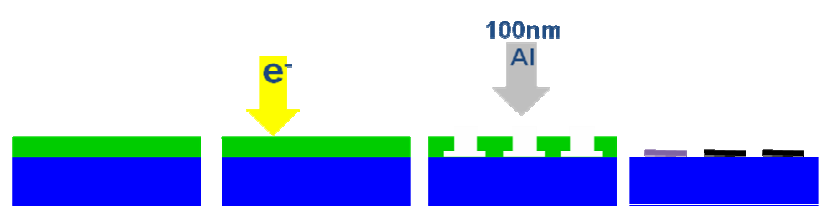

Figure 2. Process steps for e-beam processing of SAW resonators: Spin-off of several PMMA layers, e-beam lithography and developping, physical vapor deposition of aluminium and lift-off of the PMMA.

a)

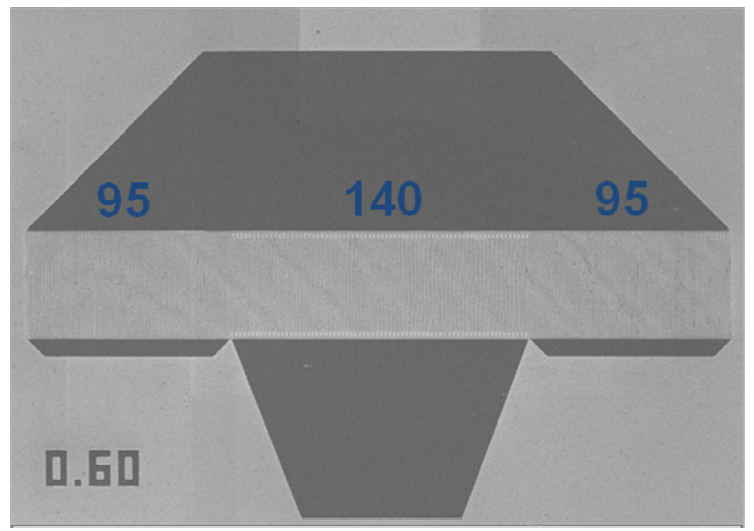

b)

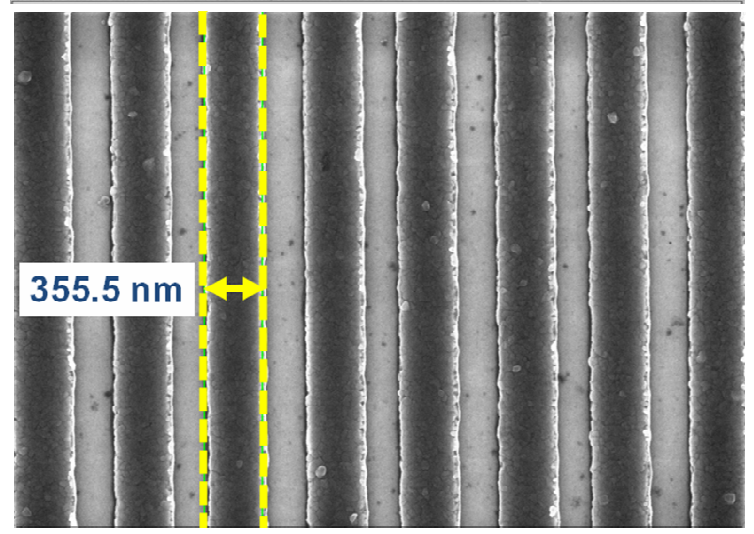

Figure 3. SEM image of a fabricated SAW synchronous resonator with a metallization ratio $\mathrm{a} / \mathrm{p}$ of 0.6 , a digital transducer with 140 fingers and reflectors on both left and right side, with 95 fingers each.

(a) Overview of resonator, (b) Close-up view of IDT.
A layered resist system with several PMMA layers of different thicknesses was used to achieve a T-shape profile for the lift-off processing. PMMA was used because of its properties, such as: high resolution, and high contrast positive tone resist for e-beam lithographic processes. Images are formed by the photo scission of the polymer backbone, which reduces the molecular weight and increases the developer solubility of the exposed resist. A 4\% 600k (molecular weight) PMMA resist from Allresist was used for the first two layers. A higher molecular weight PMMA resist was used for the third layer. A soft bake of every layer was performed after each spin-off. A standard MicroChem developer with 1:3 ratio of MIBK and IPA was used. A descum plasma process was applied to ensure a clear substrate surface of the opened areas.

Finally, a $100 \mathrm{~nm}$ thick aluminum layer was physically vapor deposited, and a lift-off process was performed overnight using acetone.

Several wafers with resonators were produced on a-plane $\mathrm{GaN}$ with a $1 \mu \mathrm{m}$ thin-film thickness. SEM photographs of a resonator are shown in Figure 3. The relatively thin $\mathrm{GaN}$ thickness of $1 \mu \mathrm{m}$, in relation to the sapphire substrate of $500 \mu \mathrm{m}$, guaranteed a low wafer bow resulting in a higher processibility and reproducibility of the resonators.

\section{MEASUREMENT AND SIMULATION}

\subsection{Harmonic Admittance}

Harmonic admittances of interdigital transducers on top of a-plane $\mathrm{GaN}$ were computed using the Blötekjaër approach [1] with constants found in the literature [8], [9]. For the determination of the polarization of the acoustic waves, the displacement amplitudes of the harmonic admittance were determined. By changing the orientation of the IDTs, different wave types can be observed in a-plane GaN. The reported curves correspond to surface waves.

\subsection{Results}

The 2D computation in the frequency range of 3 to 5 $\mathrm{GHz}$ of the device on top of $1 \mu \mathrm{m}$ a-plane GaN shows two strong resonance modes. The first mode is at $\mathrm{f}_{0}=$ $3.44 \mathrm{GHz}$ and the second mode at $\mathrm{f}_{1}=4.27 \mathrm{GHz}$, as shown in the upper plot of Figure 4. The measurements of fabricated devices (lower plot in Figure 4. ) show several modes at frequencies $\mathrm{f}_{0}=3.09 \mathrm{GHz}, \mathrm{f}_{1}=4.18$ $\mathrm{GHz}$ and $\mathrm{f}_{2}=4.81 \mathrm{GHz}$. The observed frequency difference of nearly $400 \mathrm{MHz}$ between simulation and measurement is due to errors in the bulk material data used for simulation [7], and errors in the material data of MOVPE processed crystalline thin films, which had a number of stacking faults and inhomogeneous varying area restraint [8]. 
a)

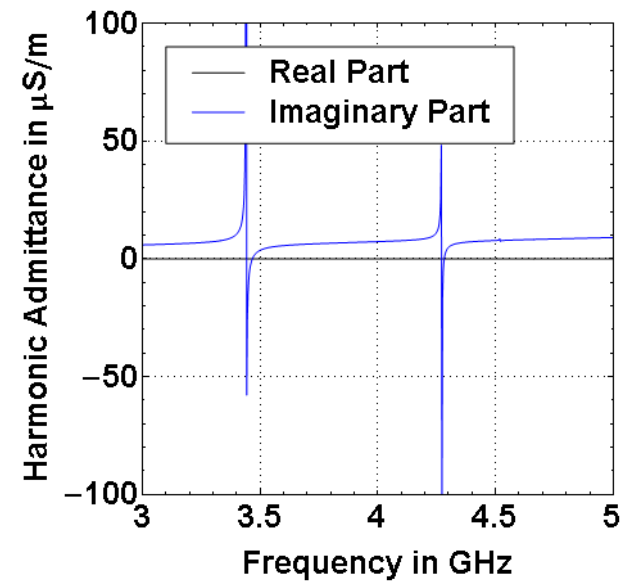

b)

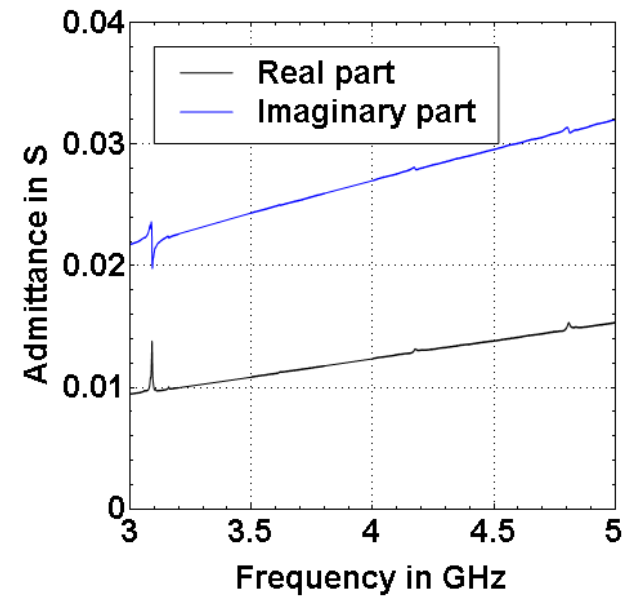

Figure 4. 2D simulation $(a)$ and measurement $(b)$ of IDT on $1 \mu \mathrm{m}$ a-plane $\mathrm{GaN}$.

(a) Simulated modes $\mathrm{f}_{0}=3.44 \mathrm{GHz}$ and $\mathrm{f}_{1}=4.27 \mathrm{GHz}$, (b) Measured modes $\mathrm{f}_{0}=3.09 \mathrm{GHz}, \mathrm{f}_{1}=4.18 \mathrm{GHz}$, and $\mathrm{f}_{2}=4.81 \mathrm{GHz}$.

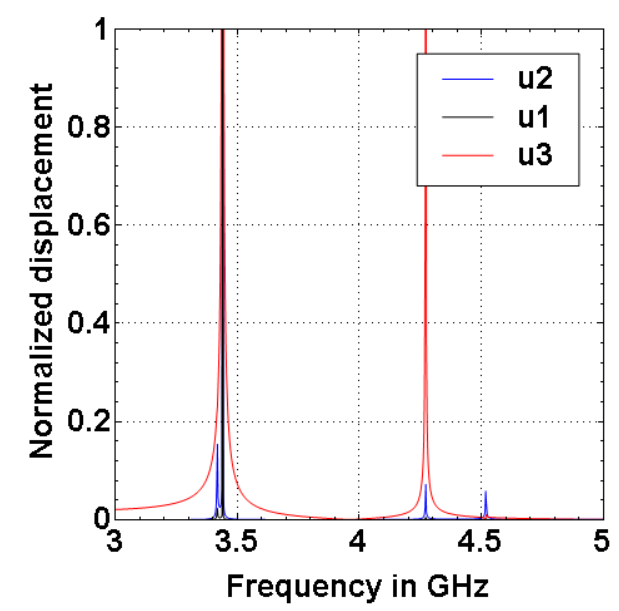

Figure 5. Normalized displacement of a 2D simulation of an infinite IDT on a $1 \mu \mathrm{m}$ a-plane GaN.

$\mathrm{u}_{1}=$ parallel, $\mathrm{u}_{2}=$ vertical, $\mathrm{u}_{3}=$ horizontal to the wave propagation direction.

$\mathrm{u}_{1}$ and $\mathrm{u}_{2}$ : elliptic contribution, $\mathrm{u}_{3}$ : shear component.
The wave polarizations of the different modes were extracted from periodic FEM/BEM simulations. The results for a $1 \mu \mathrm{m}$ a-plane $\mathrm{GaN}$ thin film are shown in Figure 5. , corresponding to the harmonic admittance shown in Figure 4. Both modes exhibit strong horizontal shear $\left(u_{3}\right)$ wave characteristics, but only the first mode has been clearly observed in the measurements.

\section{TEMPERATURE SENSITIVITY}

The temperature coefficients of frequency were measured using a Digit Concept thermal chuck on a Cascade Summit probe station over a temperature range from room temperature to $200^{\circ} \mathrm{C}$. For frequency analysis, the first three modes were measured with an E5071B Agilent Technology network analyzer..

As shown in Figure 6. the synchronous SAW resonators exhibit negative temperature coefficients of frequency of about $30 \mathrm{ppm} / \mathrm{K}$.

\section{CONCLUSIONS}

Surface acoustic wave devices on a-plane GaN thin films were fabricated using e-beam lithography and then characterized.

The temperature coefficients of frequency on a-plane oriented thin films were measured. The measurements and simulation results for SAW devices on a-plane oriented thin films differ by several hundreds of $\mathrm{MHz}$, due to still unknown material parameters of our gallium nitride thin films. Future work will focus on extracting and measuring material parameters of the gallium nitride thin films, to improve the match between the simulations and the measurements, as well as working with other MOVPE grown a-plane oriented piezoelectric III-V compound semiconductors, like aluminum nitride.

\section{ACKNOWLEDGMENT}

Thanks to Dr. Bert Nägel and Dr. Sandra Wolff from the University of Kaiserslautern, Germany, for performing the electron beam lithography. 

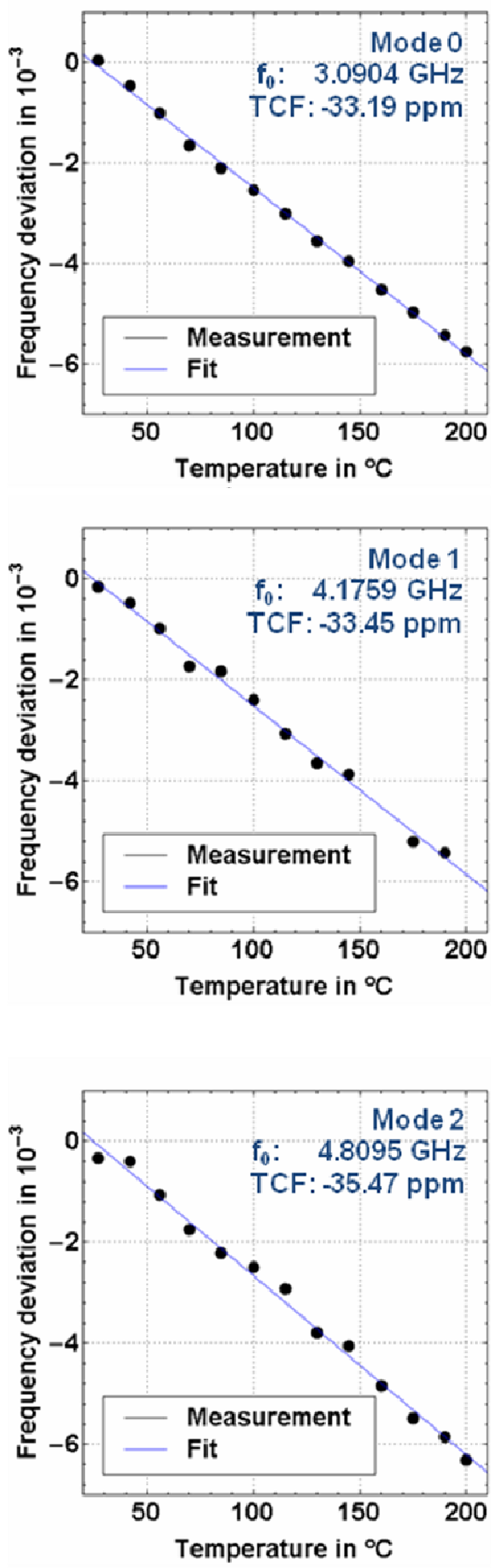

Figure 6. Measurement curves of frequency deviation in dependancy of temperature change of a synchronous SAW resonator on $1 \mu \mathrm{m}$ thick a-plane GaN.

\section{REFERENCES}

[1] M.G. Rosenfield, M.G.R. Thomson, P.J. Coane, K.T. Kwietniak, J. Keller, D.P. Klaus, R.P. Volant, C.R. Blair, K.S. Tremaine, T.H. Newman, F.J. Hohn, "Electron-beam lithography for advanced device prototyping : process tool metrology", Journal of vacuum science \& technology. B. Microelectronics and nanometer structures. Processing, measurement and phenomena, vol. 11, no. 6, pp. 2151-3015, 1993.

[2] A.A. Tseng, Kuan Chen; C.D Chen, K.J. Ma, "Electron beam lithography in nanoscale fabrication: recent development," Electronics Packaging Manufacturing, IEEE Transactions on, vol.26, no.2, pp. 141-149, Apr. 2003.

[3] G.S. Gatlin, C.W. Eskridge, J.M. Brinkerhoff, J.D. Fife, J.R Jessing, "Process development of electron beam lithography in an academic environment," Microelectronics and Electron Devices, 2004 IEEE Workshop on, pp. 117-119, 2004.

[4] M.N. Yoder, "Gallium nitride past, present, and future," High Speed Semiconductor Devices and Circuits, 1997. Proceedings., 1997 IEEE/Cornell Conference on Advanced Concepts in, pp. 312, Aug. 1997.

[5] S.V. Pettersen, T. Tybell, A. Ronnekleiv, S. Rooth, V. Schwegler, J.K. Grepstad, "Surface Acoustic Wave Resonator From Thick MOVPE-Grown Layers of GaN(0001) on Sapphire", Mat. Res. Soc. Symp. Proc., Electronic and Photonic Materials, vol. 743 , L6.37, 2002.

[6] K. Nishimura, N. Shigekawa, H. Yokoyama, M. Hiroki, K. Hohkawa, "SAW characteristics of GaN layers with surfaces exposed by dry etching", IEICE Electron. Express, Vol. 2, No. 19, pp. 501-505, 2005.

[7] K. Blötekjaër, K.A. Ingebrigsten, H. Skeie, "A method for Analysing Waves in Structures Consisting of metallic Strips on Dispersive Media", IEEE Trans. on Electron. Devices, Vol. 20, pp. 1133-1138, 1973.

[8] O. Ambacher, J. Majewski, C. Miskys, A. Link, M. Hermann, M. Eickhoff, M. Stutzmann, F. Bernardini, V. Fiorentini, V. Tilak, B. Schaff and L.F. Eastman, "Pyroelectric properties of $\mathrm{Al}(\mathrm{In}) \mathrm{GaN} / \mathrm{GaN}$ hetero- and quantum well structures", J. Phys.: Condens. Matter, vol. 14, pp. 3399-3434, 2002.

[9] A. Krost, A. Dadgar, G. Strassburger, R. Clos, "GaN-based epitaxy on silicon: stress measurements", phys. stat. sol. (a), vol. 200, no. 1, pp. 26-35, 2003. 